\title{
Electron Beam Welding
}

\author{
by Clyde M. Adams, Jr. of Massachusetts Institute of Technology
}

\begin{abstract}
The electron beam is fast gaining recognition as a unique, highvacuum heat source for processing materials ... finding application in the drilling, cutting, refining, and welding of metals and non-metals ... and showing promise in coating, cladding, and casting. First applied to welding by $K$. Steigerwald of Carl Zeiss in West Germany about 1950 , several US firms are currently developing and manufacturing electron beam welding equipment.

The electron beam is unique as a processing heat source by virtue of its intensity (over $10,000^{\circ} \mathrm{F}$ ), focusing ability, and high vacuum ( 0.03 to $0.05 \mu)$ operation. This combination clearly distinguishes the electron beam from arc, induction, or resistance heating. Before the electron beam, there was no means of performing conventional fusion welding in a high vacuum with a moving heat source-the closest approach being dry-box equipment in which an arc is sustained in a chamber filled with purified inert gas. In this sense, many regard the electron beam as the ultimate dry box.
\end{abstract}

\section{Operation}

The principle of operation is that of the cathode-ray tube, wherein a heated tungsten filament is the cathode from which emitted electrons are accelerated toward an anode, using a potential difference of 5000 to $150,000 \mathrm{v}$. In some welding equipment, the anode is the workpiece itself, an arrangement common to many zone-refining or drip-melting operations. However, recent equipment has incorporated an integral ring-shaped anode through which most of the electrons are accelerated, and after leaving the region of steep potential gradient they travel at constant velocity toward the workpiece. The tendency for the beam to diverge electrostatically after passing through the anode is overcome by electromagnetic or electrostatic focusing. The workpiece and the anode are usually at the same (ground) potential.

There are two major reasons for having the anode separate from the workpiece: 1) filament life is improved, not only because the filament is geometrically remote from the workpiece-which is the primary source of contamination-but also because there is no electrostatic field to accelerate the positive ions produced by bombardment of the workpiece toward the filament; and 2) operative control is improved since the electron beam can be directed within recesses or re-entrant angles in the workpiece without seeking the shortest path.

There is also a choice to be made as to the type of focusing employed. Electromagnetic focusing accords some advantages in circuitry and control, but for high-power operation, the focusing coils are necessarily somewhat massive and require water cooling. In electrostatic focusing, a secondary circuit is required to maintain the relatively low positive-potential of the focusing apertures, but these apertures may be of light construction and render more feasible the movement of the gun for automatic welding within the vacuum chamber.

Joint preparations and welding procedures are still somewhat primitive, primarily because automatic wire-feed mechanisms have not been incorporated into electron beam equipment. To date, simple co-fusion of the members to be joined has been the rule with filler, if any, being first placed along the intended seam. There is no reason to doubt that cold feed metal techniques eventually will be applied to electron beam welding, although more quantitative information on the over-all thermal characteristics of the beam as a moving heat source must precede the evolution of firm welding procedures.

There is some controversy as to the optimum voltage range for electron beam equipment. For a given power input, the higher the voltage, the narrower is the heat source and the deeper the penetration. Thus, high voltage looks attractive for drilling, cutting, or joining of thick members. (The deep, narrow fusion zones produced at very high voltages bear some similarity to brazed joints.) At the other extreme, relatively low potentials $(5,000$ to $10,-$ $000 \mathrm{v}$ ) can be used quite successfully for refining operations in which focusing is relatively unimportant. Weld fusion zones of shapes similar to those produced in permanent electrode arc welding are obtained in the range of 15,000 to $30,000 \mathrm{v}$ and 1 to $8 \mathrm{kw}$. For welding, the low- voltage equipment is attractive from several standpoints: 1) low-cost power supply, 2) short wave-length $\mathrm{X}$-rays are not produced, and 3 ) equipment and insulation are substantially less massive. The governing technical relationship in selecting voltage is that the minimum beam cross-section (in the focused region) is proportional to beam amperage, so that high power and narrow focusing can be achieved only at high voltage. However, in the many applications in which fusion zone widths of 0.050 to 0.150 in. are tolerable in stock thicknesses of less than 0.300 in., potentials as low as $15,000 \mathrm{v}$ will find application.

\section{Applications}

The intensity of the heat source and the purity of the environment have directed much current attention to welding reactive and refractory metals. In addition, the process deserves attention for application to ultra-high-strength steels in which the complete absence of hydrogen is so essential to the consummation of a successful weld. Some difficult pneumatic problems have been solved using the electron beam welder for encapsulation; in fact, the biggest current commercial application is in sealing nuclear reactor fuel elements. Many of the most attractive characteristics have yet to be exploited. For example, by defocusing, the beam can be used for pre- or post-heat treatment in association with welding and offers much potential for vacuum brazing.

The future of the electron beam for welding is definitely not confined to the laboratory. Size is no real limitation, since large vacuum chambers have become virtually commonplace, and, in fact, a large vacuum reservoir is an advantage in electron beam welding where outgassing is likely to be irregular. It is of interest that the process is fail-safe against accidental weld contamination, since the electron beam becomes inoperative at pressures above $1 \mu$. When more utilitarian and maneuverable equipment is developed, the electron beam will become a serious contender for many critical joining applications in which the refining and high intensity inherent to the process can offer any advantage. 